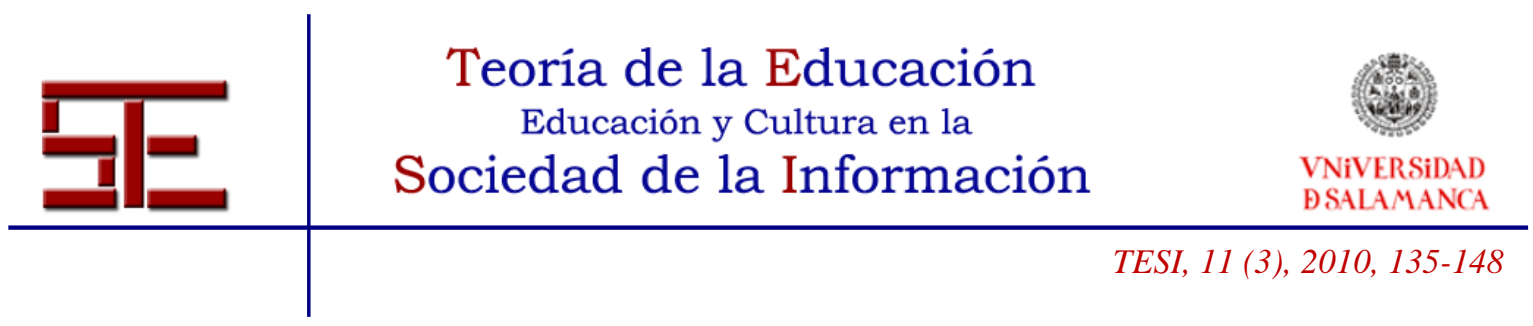

\title{
CAMBIO DE ACTITUDES Y VALORES ANTE LA ENERGÍA TRAS EL USO DE UN JUEGO DE ROL
}

Resumen: Los juegos de rol en educación se han definido como dramatizaciones que se vertebran en torno a una situación concreta y utilizan la asignación de papeles a alumnos, quienes deberán defender con argumentos una opinión (previamente perfilada) frente a un problema. Cada vez más utilizados en la educación formal y no formal, destacan entre sus ventajas el convertir lo lúdico en aprendizaje, potenciar el aprendizaje de actitudes y valores y la conexión que se realiza con la vida real entre otros.

La utilización de un juego de rol en una investigación educativa sobre cambio de actitudes y valores ante los recursos energéticos cuyos resultados educativos fueron positivos, permitió repetir dicha investigación usando la variable "utilización/no utilización del juego de rol", lo que concedió la posibilidad de analizar la importancia de los juegos de rol en la potenciación de cambios en las actitudes y valores de los usuarios de estos juegos.

Palabras clave: juegos de rol; actitudes; valores; educación no formal; autosuficiencia energética; energías alternativas.

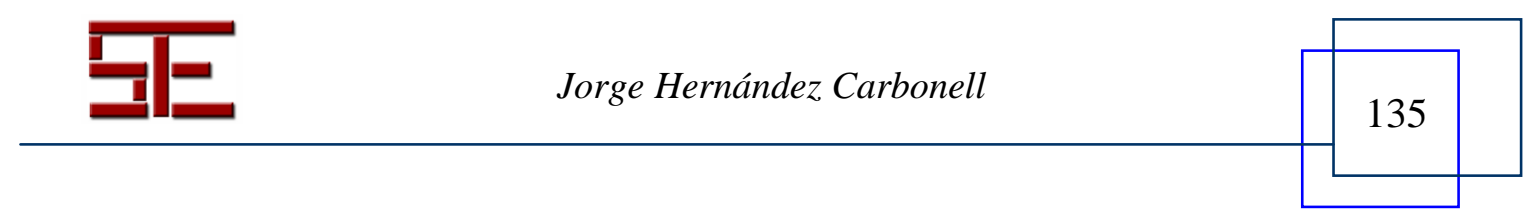




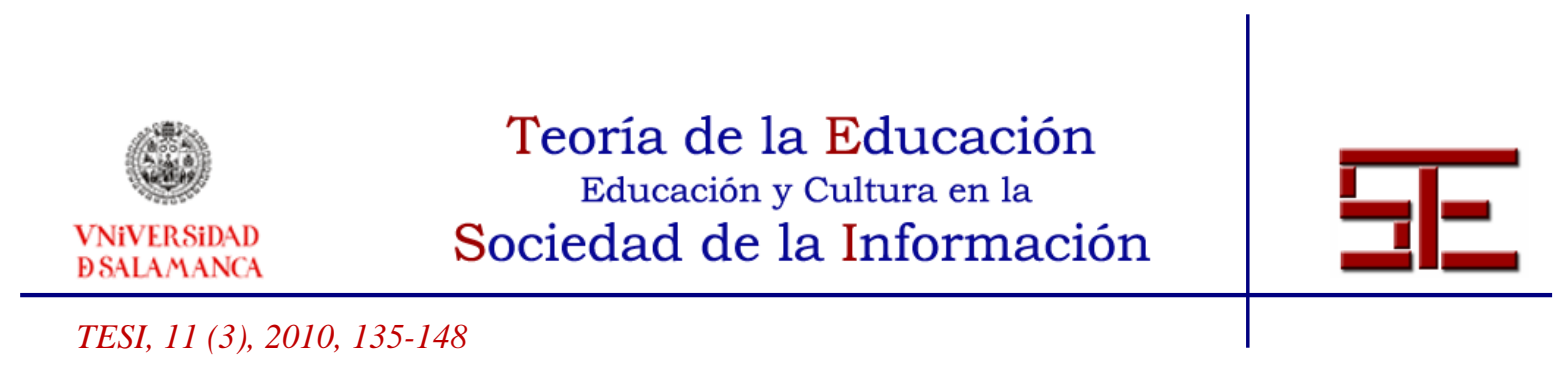

\title{
"CHANGE OF ATTITUDES AND VALUES BEFORE THE ENERGY AFTER THE USE OF A ROLE-PLAYING GAME"
}

\begin{abstract}
The role-playing game in education have been defined as dramatizations that are carry out around a situation makes specific and use the allocation of papers to students, who will have as opposed to, to defend with arguments an opinion (previously outlined) a problem. More and more used in the formal and non formal education, they emphasize between its advantages turning the playful thing learning, harnessing the learning of attitudes and values and the connection that is made with the real life among others.

The use of a role-playing game in an educative investigation on change of attitudes and values before the power resources whose educative results were positive, allowed to repeat this investigation using the variable "utilization/no use of the role-playing game", which granted the possibility of analyzing the importance of the role-playing game in the involution of changes in the attitudes and values of the users of these games.
\end{abstract}

Key words: role-playing game; attitudes; values; non formal education; power selfsufficiency; alternative energies.

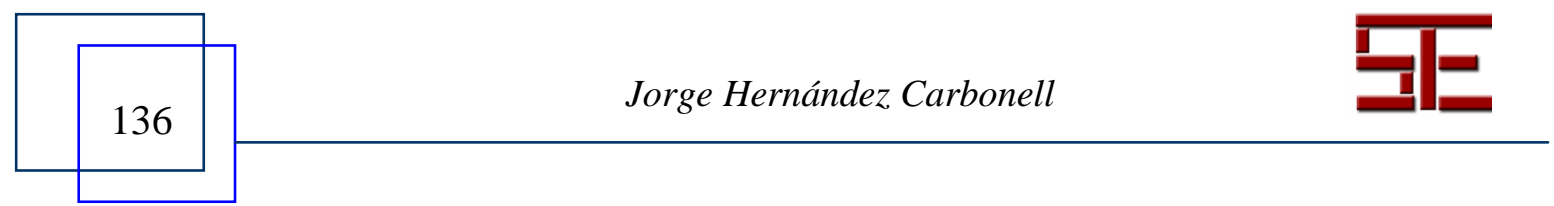




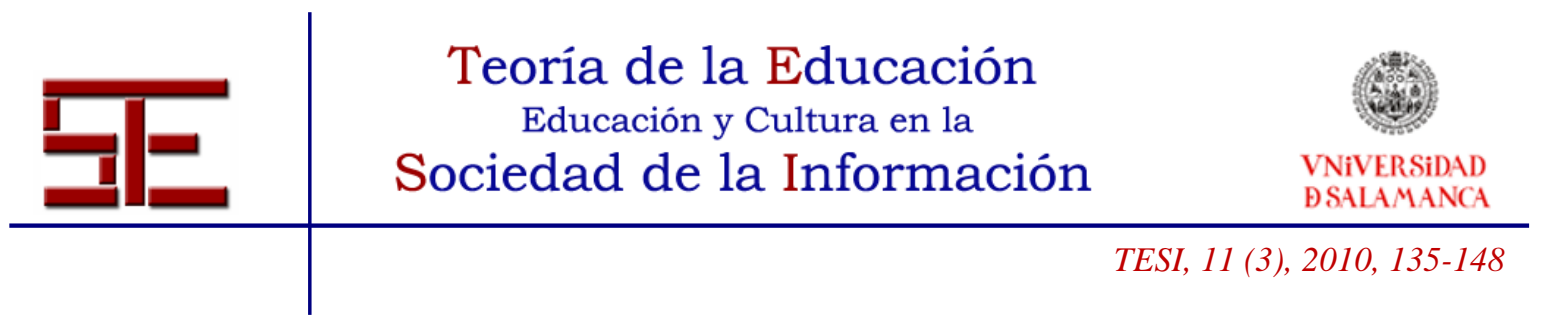

\section{CAMBIO DE ACTITUDES Y VALORES ANTE LA ENERGÍA TRAS EL USO DE UN JUEGO DE ROL}

Fecha de recepción: 07/04/2010; fecha de aceptación: 01/09/2010; fecha de publicación: 30/11/2010

Jorge Hernández Carbonell

jorgeh0@hotmail.com

Universidad de Valencia

\section{1.- INTRODUCCIÓN}

Los juegos de rol en educación se han definido (Caurin, 1999) como dramatizaciones que se vertebran en torno a una situación concreta y utilizan la asignación de diversos papeles a diferentes grupos de alumnos, los cuales deberán defender con argumentos una opinión (previamente perfilada) frente a un problema. Son cada vez más utilizados tanto en la educación formal como en la educación no formal, destacándose entre sus ventajas el convertir lo lúdico en aprendizaje, potenciar el aprendizaje de actitudes y valores y permitir orientar al profesor sobre una estrategia didáctica a seguir, ya que afloran errores conceptuales, procedimientos y actitudes que puedan ser corregidos y modificados. Así mismo, motivan al alumno, destacan por su simplicidad y abstracción y permiten una conexión con la vida real que otras formas de aprendizaje no logran (García y Nando, 2000).

En el año 2000, y a raíz de un estudio encargado por la Oficina del Defensor del Menor al Colegio Oficial de Psicólogos de Madrid con el fin de incrementar el conocimiento de este tipo de juegos y, sobre todo, de aportar evidencia empírica sobre los efectos de los juegos de rol en el desarrollo evolutivo de los jóvenes que los practican, aparece el estudio Efectos psicosociales de los juegos de rol en el desarrollo social y cognitivo de los menores (Esteban y Vecina, 2000) donde se señala que "La eliminación de estereotipos negativos y el énfasis en sus rasgos positivos permitiría convertir los juegos de rol en una valiosa herramienta de intervención social con adolescentes para la promoción activa de actitudes y conductas positivas relacionadas con la tolerancia, la coeducación, la educación para la paz y la prevención de conductas asociales, etc.

La utilización de un juego de rol en una investigación educativa sobre cambio de actitudes y valores ante los recursos energéticos, cuyos resultados educativos fueron

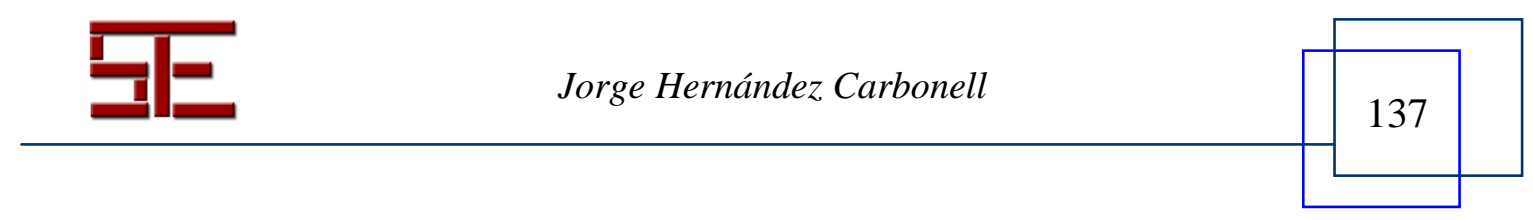




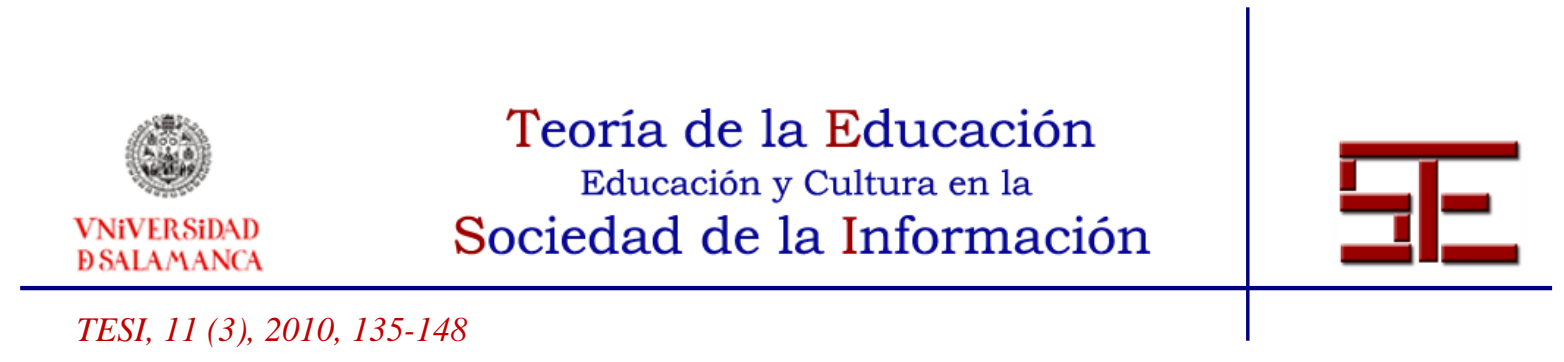

positivos, permitió la posibilidad de repetir dicha investigación usando la variable "utilización/no utilización del juego de rol", lo que nos concedería la posibilidad de analizar la importancia de los juegos de rol en la potenciación de cambios en las actitudes y valores de los usuarios de estos juegos.

\section{2.- JUEGO PROPIAMENTE DICHO}

En la presente investigación se utilizó un juego de rol de creación propia, ideado para dicha investigación.

El juego en cuestión consiste en la simulación de un debate entre los principales representantes de los diferentes estamentos políticos y sociales de las ciudades de una isla que busca la autosuficiencia energética. Se asignan papeles de forma equilibrada, de manera que haya tantos alumnos a favor de cualquiera de las diferentes energías posibles. Aparecen también papeles como: vecinos, científicos, asociaciones locales, etc., consiguiendo de esta manera diversidad de puntos de vista. Se proponen distintas opciones energéticas, tanto renovables como no renovables, y energética que considera más idónea como la localización de dicha opción energética, o en su caso, dar las razones por las que no situarlas en una zona determinada y defender su postura ante la postura de los otros participantes.

A cada alumno se le proporciona un juego de fichas donde se explica de forma resumida cada tipo de energía que es posible poner en la isla, con su coste y sus ventajas e inconvenientes, así como un rol (en forma de ficha) que personaliza uno de los representantes políticos o sociales de la isla y que han de interpretar.

El juego se desarrolla durante una semana de estancia en el CEMACAM Los Molinos. En dicho lugar se trabajarán las actitudes, valores y conocimientos ante las energías alternativas, sirviendo el juego de rol de nexo de unión para la adquisición de dichos parámetros. Antes del comienzo de las actividades y después de la finalización de las mismas, los alumnos completan una encuesta de preguntas abiertas que nos permitirán conocer sus actitudes ante las energías alternativas y otros parámetros que nos permitirán analizar la evolución de los mismos tras la realización de las diferentes actividades. Así, el juego de rol se propondrá, en aquellos grupos que lo realicen, al comenzar la estancia en el centro y se desarrollará durante ésta hasta el último día de la semana, día en el que se realizará la actividad central del juego de rol de exposición de opiniones de cada grupo, abriéndose un debate en el que cada representante político y/o social ha de proponer y defender su postura ante el problema planteado.

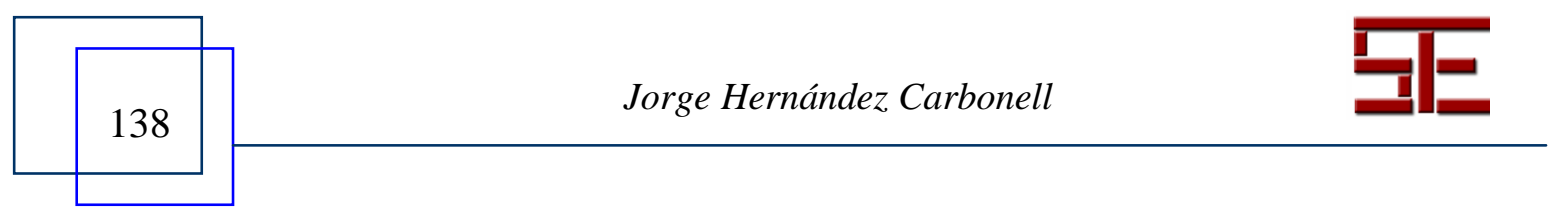




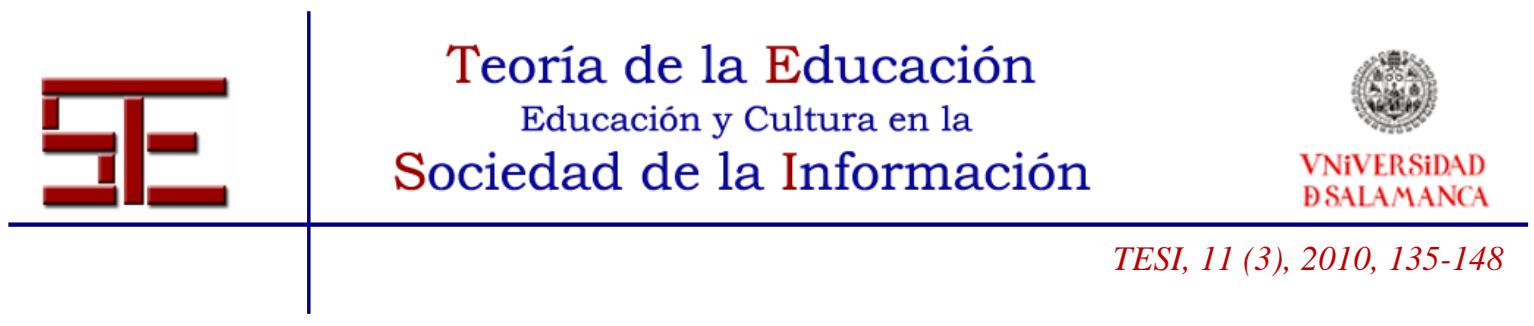

\section{METODOLOGÍA Y DISEÑO DE LA INVESTIGACIÓN}

\section{1.- Diseño general}

El presente trabajo plantea la posibilidad de comprobar que la utilización de un juego de rol provocará cambios en las actitudes y valores de los jugadores, cambios que podían después ser aumentados posteriormente con actividades dirigidas a potenciar dichos valores y actitudes.

En un trabajo inicial (Hernández, 2005) se comprobó que, tras la estancia en un Centro de Educación Ambiental (el Centro de Educación Medio Ambiental Los Molinos de la Caja de Ahorros del Mediterráneo) trabajando como tema central el problema energético, la siguiente hipótesis era correcta:

"Si las actividades que fundamentan y motivan el cambio de actitudes y valores ante los problemas energéticos se integran de manera intensiva, dentro de las actividades diarias, es posible producir dicho cambio en un espacio corto de tiempo, si en los alumnos existe una predisposición motivacional y preconceptos positivos".

Comprobada esta hipótesis (Hernández, 2005), se acometió la investigación de analizar la importancia que tenía el uso del juego de rol utilizado en dicha investigación para la consecución de cambio de actitudes y valores.

Para ver si era correcta esta hipótesis, se realizaron las mismas actividades que nos habían permitido producir cambio de actitudes y valores ante los problemas energéticos en un espacio corto de tiempo, siendo la variable utilización de un juego de rol la única que era diferente, lo que nos permitiría ver la importancia de dicha utilización para la consecución de dichos cambios en actitudes y valores.

\section{2.- El juego de rol en el global de las actividades}

Debido a que el cambio de actitudes y valores es lento y costoso al tener que ser internalizadas por los estudiantes, se estudió el cambio producido con el juego de rol en tan sólo cinco actitudes principales y un valor principal, que coincidían con las estudiadas en el CEMACAM Los Molinos a lo largo de la semana de estancia. Estas actitudes fueron:

- Actitud de defensa del medio ambiente con argumentos fundamentados y contrastados.

- Actitud crítica ante problemas y situaciones ambientales energéticas.

- Actitud ante la limitación de los recursos energéticos.

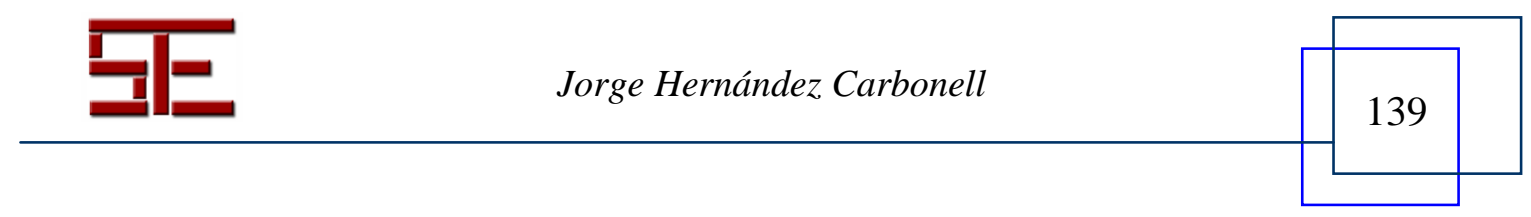




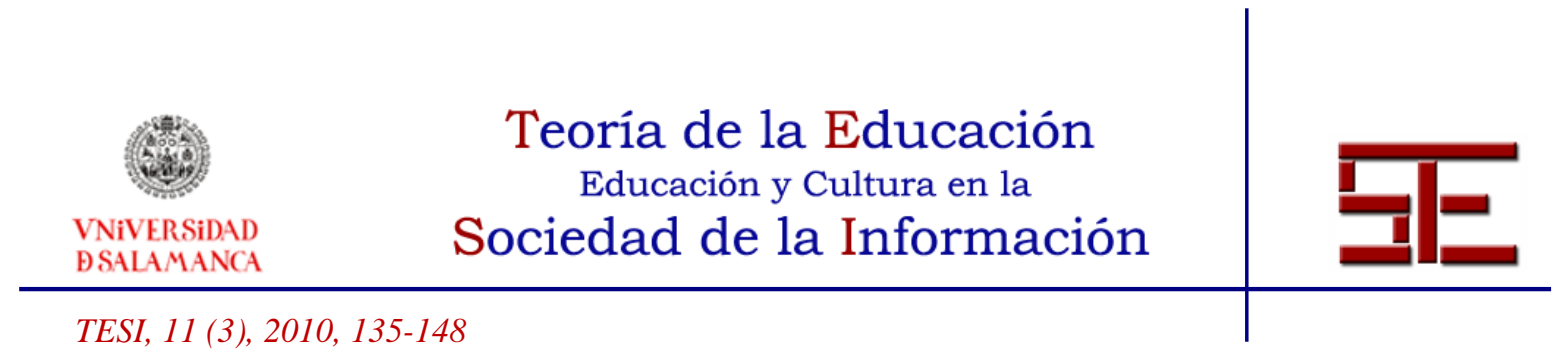

- Actitud de ahorro energético.

- Actitud positiva ante las energías alternativas.

En lo referente a los valores, el objetivo primordial que pretendimos obtener era:

- Modificar o fomentar los valores de los estudiantes hacia un modelo energético sostenible, capaz de combinar en la medida de lo posible el actual desarrollo con el respeto al medio ambiente.

\section{3.- Cuestionarios utilizados}

Para realizar la investigación, decidimos confeccionar cuestionarios con preguntas de carácter abierto. Estos cuestionarios, ya utilizados por diferentes autores para la medición de actitudes (Escudero, 1985; Aikenhead, 1988; Guilbert \& Meloche 1993; Acevedo, 2000 ; Caulín, Gil ; Llopis, 2000) tienen la cualidad de implicar al estudiante en la respuesta, lo cual nos permitirá inferir las actitudes y valores a estudiar al no ser directamente observables (Caurín, 1999). Dichos cuestionarios fueron contestados en dos ocasiones por los mismos grupos: la primera, antes de iniciar la investigación (Primer día de la estancia en el CEMACAM Los Molinos) y la última, tras finalizar la estancia y todas las actividades (Último día de la estancia en el CEMACAM Los Molinos).

El cuestionario era el siguiente Nombre:

Edad:
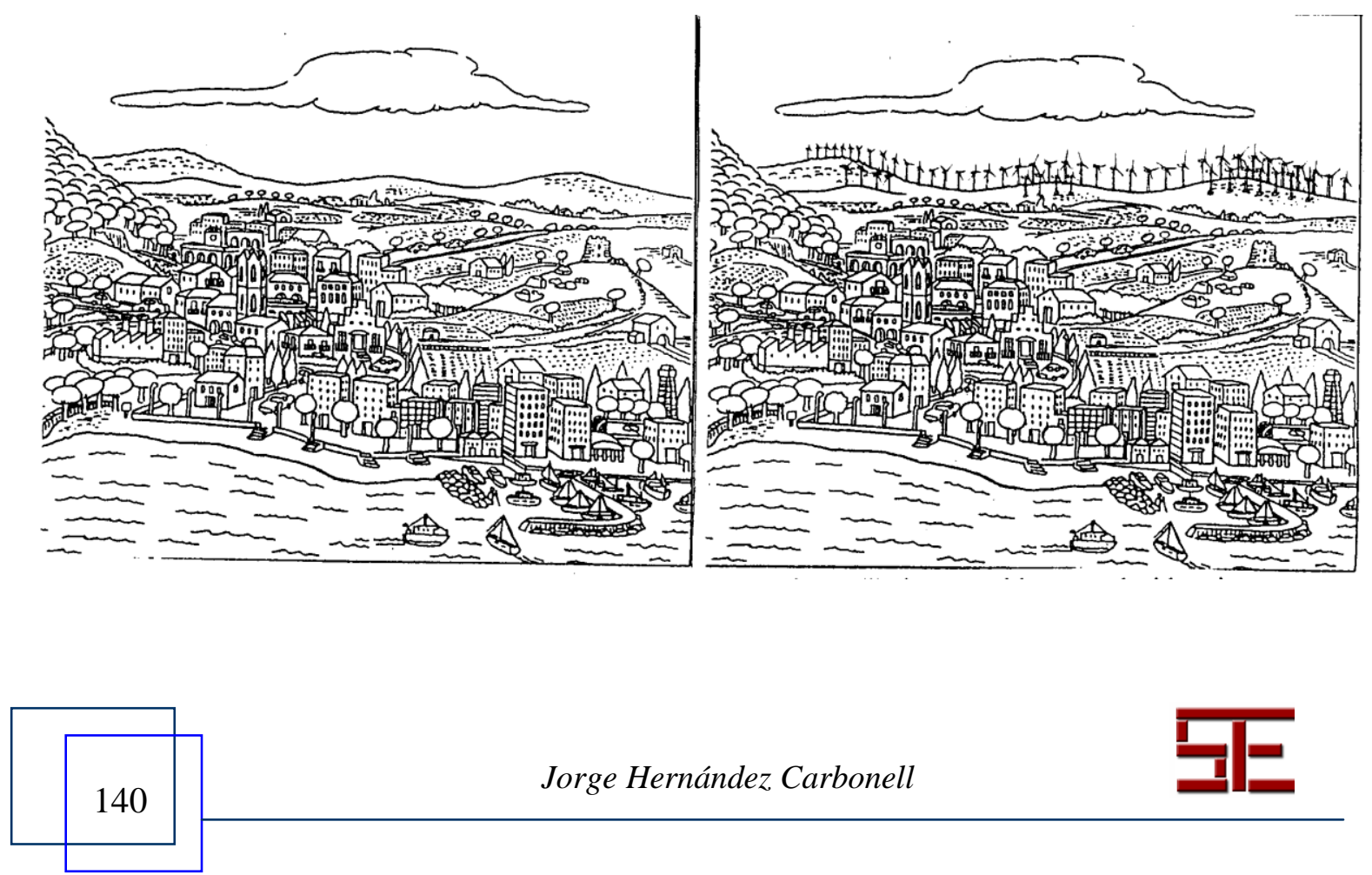


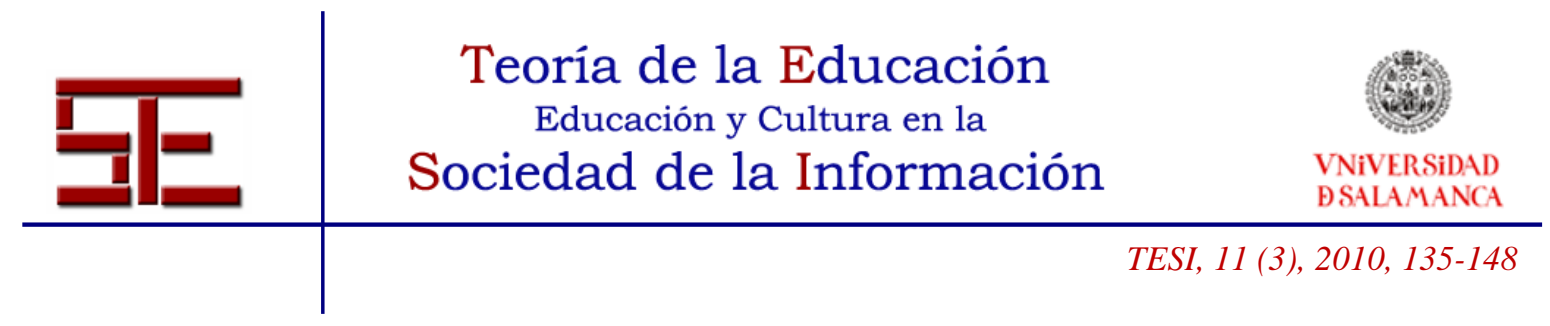

1. Imagínate que eres el Alcalde del pueblo del primer dibujo y te piden que decidas si instalar una instalación eólica situada en la montaña que hay encima del pueblo (como en el segundo dibujo). Las ventajas de su instalación serían que tendríais electricidad muy barata y no contaminaría el medio ambiente. La desventaja es que algunos sectores del pueblo piensan que eso afea el paisaje, lo cual puede alejar al turismo que va a esas montañas, que es la principal fuente de dinero de la zona.

¿Qué harías? ¿Por qué?

2. Eres el responsable de proporcionar electricidad a unas casas aisladas. Una vez realizados los estudios necesarios, descubres que sólo existe una forma de llevar la electricidad a esas casas: pasar un tendido eléctrico que cruce una zona natural de gran belleza. Tienes que tomar una decisión:

- Respetar la zona natural (con los problemas que ocasionaría a los habitantes de las casas aisladas),

- Pasar el tendido eléctrico por la zona natural (con los problemas que ocasionaría en la zona natural) o

- Buscar una alternativa a estas dos (deberás explicarla) ¿Qué decisión tomas?

3. Imagínate que después de ver una hora la televisión te dicen que tienes que pagar la electricidad gastada. ¿Piensas que deberías pagar lo mismo si te dijeran que esa electricidad proviene de una central nuclear o de una placa solar? Si la respuesta es que no se debería pagar lo mismo, indica por cuál se debería pagar más y explica por qué.

4. Se dice que hay que apagar las luces cuando no se utilizan para ahorrar energía, pero teniendo en cuenta la cantidad de gente que no lo hace, ¿por qué piensas que deberías hacerlo tú?

5. Los países occidentales (del "primer mundo") consumen mucha más energía que los países del "tercer mundo". ¿De quién piensas que es esto culpa? ¿Cómo solucionarías tú esta situación?

\section{4.- Diferentes colegios}

La muestra utilizada para realizar el tratamiento fue de 396 jóvenes pertenecientes a 9 colegios públicos y concertados urbanos de igual nivel educativo $\left(2^{\circ}\right.$ de ESO) y distintas zonas geográficas que realizaron una estancia de una semana para trabajar el tema de las

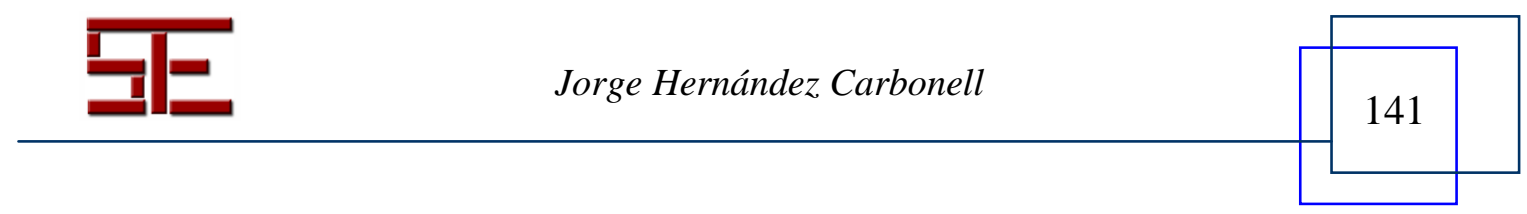




\begin{tabular}{ccc}
$\begin{array}{c}\text { Teoria de la Educación } \\
\text { Educación y Cultura en la } \\
\text { VNiVERSIDAD } \\
\text { DSALAMANCA }\end{array}$ & Sociedad de la Información \\
\hline TESI, $11(3), 2010,135-148$ &
\end{tabular}

energías alternativas en el Centro de Educación Medio Ambiental (CEMACAM) Los Molinos. En todos ellos se realizaron todas las actividades orientadas a cambiar las actitudes y valores ante las energías alternativas, diferenciándose sólo los 5 centros del grupo A en que sí que se realizó el juego de rol frente a los 4 del grupo B que realizaron actividades sobre biodiversidad, en sustitución del juego de rol.

\begin{tabular}{|c|c|c|c|}
\hline \multicolumn{3}{|c|}{$\begin{array}{c}\text { GRUPO A: Colegios que hicieron todas las actividades incluido el } \\
\text { juego de Rol }\end{array}$} & \\
\hline GRUPO A & $\mathrm{N}$ & Centro educativo & \\
\hline Grupo 1 & 34 & IES Riba-Roja del Turia (Valencia) & Público \\
\hline Grupo 2 & 53 & IES Alquibra (Murcia) & Público \\
\hline Grupo 3 & 46 & C. P. Jorge Guillén (de Elche, Alicante), & Público \\
\hline Grupo 4 & 41 & Colegio Divino Maestro (Baza, Granada) & Concertado \\
\hline Grupo 5 & 52 & C. Santa Teresa (Alicante) & Concertado \\
\hline
\end{tabular}

\begin{tabular}{|c|c|c|c|}
\hline \multicolumn{3}{|c|}{$\begin{array}{l}\text { GRUPO B: Colegios que hicieron todas las actividades menos } \\
\text { el juego de rol }\end{array}$} & \\
\hline GRUPO B & $\mathrm{N}$ & Centro educativo & \\
\hline Grupo 1 & 45 & $\begin{array}{l}\text { C.P. Molivent (Guardamar del Segura, } \\
\text { Alicante) }\end{array}$ & Público \\
\hline Grupo 2 & 41 & C. La Inmaculada (Yecla, Murcia) & Concertado \\
\hline Grupo 3 & 48 & $\begin{array}{l}\text { C.P. José María de la Puerta (Cartagena, } \\
\text { Murcia) }\end{array}$ & Público \\
\hline Grupo 4 & 36 & C. María Auxiliadora (Alicante) & Concertado \\
\hline
\end{tabular}

\section{5.- Análisis de los datos cualitativos. Redes sistémicas}

El análisis de los datos obtenidos se concretó en cuatro niveles. El primer nivel consistió en la trascripción, traducción y codificación de los datos obtenidos de los cuestionarios. En el segundo, se creó una red sistémica (Bliss et al., 1979; Neus Sanmartí, 1989; Caurín, 1999; Sardá y Sanmartí, 2000; Bonil et al., 2004) para organizar las categorías obtenidas en el primer análisis. El tercer nivel se realizó la categorización de los distintos aspectos aparecidos en las redes sistémicas para poder medir la evolución de los sujetos. Para ello se construyeron tablas basadas en las actitudes y valores que

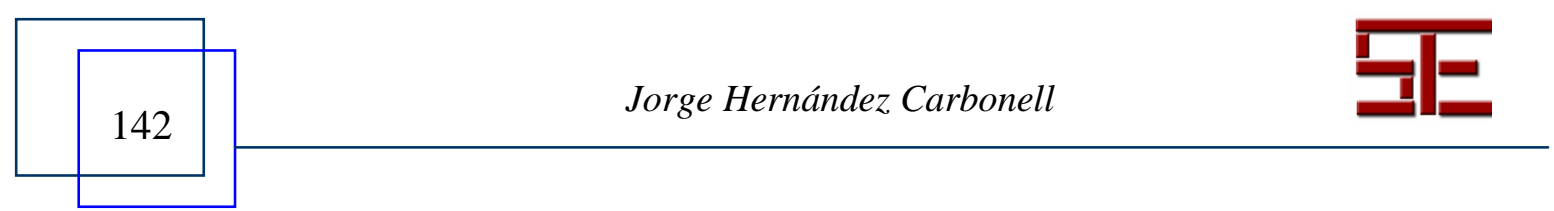




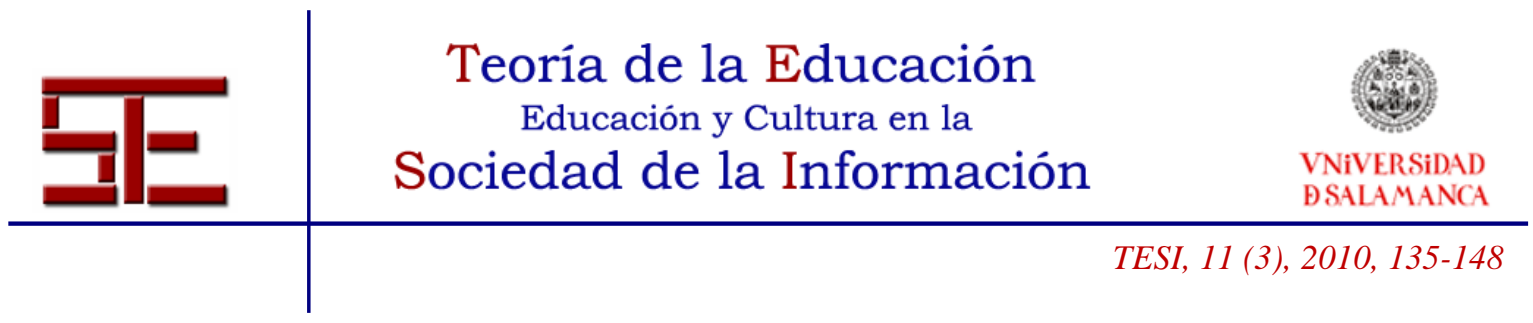

pretendíamos analizar su evolución. En dichas tablas los clasificamos según un proceso de baremación que clasifica las respuestas con un 1 o un 2, dándole el valor 1 a aquellos argumentos que iban en contra del valor o actitud que buscábamos y el valor 2 a aquellos argumentos que estaban de acuerdo del valor o actitud que buscábamos. Finalmente, en el cuarto nivel se determinó la significatividad de los datos obtenidos, realizando la prueba de chi cuadrado entre los datos obtenidos en los grupos en los que se realizo el juego de rol y los grupos en los que no se realizó. Esto nos permitió saber si existe diferencia entre realizar o no el juego de rol.

Las respuestas se clasificaron en 1 ó 2 según la siguiente baremación:

1. Argumenta motivos ambientales (Se mide en las preguntas 1, 2, 3, 4 y 5).

Valor 1. No menciona motivos ambientales o menciona motivos ambientales pero de forma no significativa, sin énfasis o apoyo explícito.

Valor 2. Menciona motivos ambientales de forma significativa, mencionando o subrayando de alguna forma la relevancia.

2. Actitud ante la limitación de recursos energéticos (Se mide en las preguntas 1, 3 y 4).

Valor 1. No menciona la limitación de recursos energéticos o menciona la limitación de recursos energéticos pero de forma no significativa, sin énfasis o apoyo explícito.

Valor 2. Menciona la limitación de recursos energéticos de forma significativa, mencionando o subrayando de alguna forma la relevancia.

3. Valora el medio ambiente como prioritario (Se mide en las preguntas 1, 2, 3 y 4).

Valor 1. No menciona el medio ambiente como prioritario o menciona el medio ambiente como prioritario pero de forma no significativa, sin énfasis o apoyo explícito.

Valor 2. Menciona el medio ambiente como prioritario de forma significativa, mencionando o subrayando de alguna forma la relevancia.

4. Actitud positiva ante las energías alternativas (Se mide en las preguntas 1, 2, 3, 4 y 5.2).

Valor 1. No menciona argumentos que indiquen una actitud positiva ante las energías alternativas o menciona argumentos que indican una actitud positiva ante las energías alternativas pero de forma no significativa, sin énfasis o apoyo explícito.

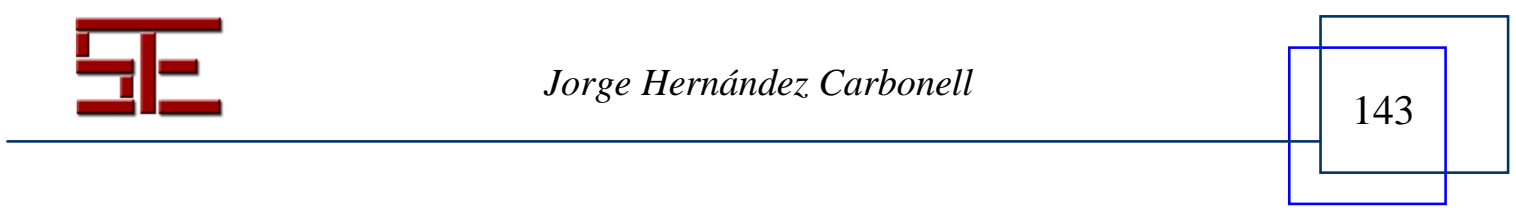




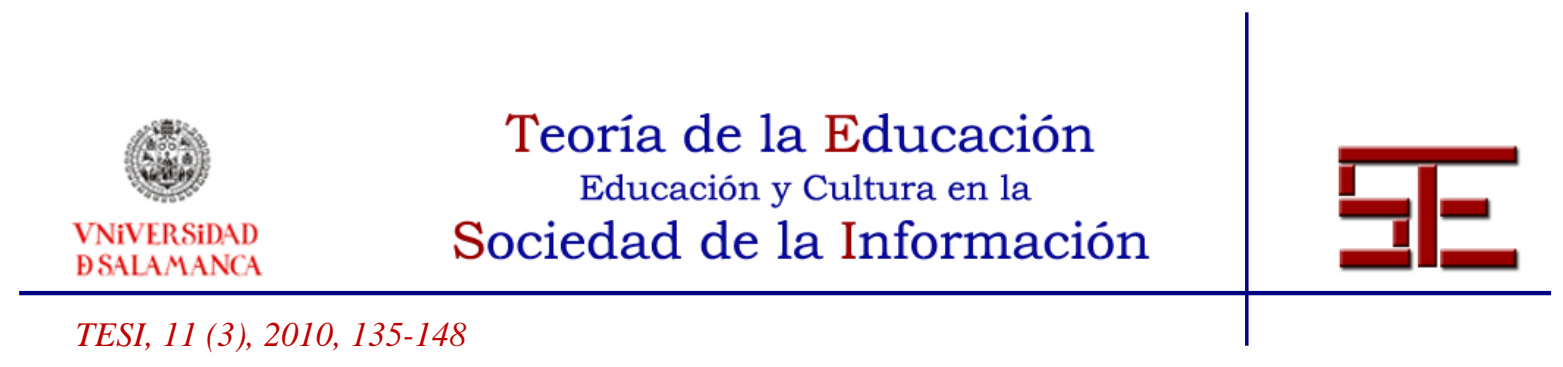

Valor 2. Menciona argumentos que indican una actitud positiva ante las energías alternativas de forma significativa, mencionando o subrayando de alguna forma la relevancia.

5. Valora el ahorro energético (Se mide en las preguntas 4 y 5.2).

Valor 1. No menciona el ahorro energético o menciona el ahorro energético pero de forma no significativa, sin énfasis o apoyo explícito.

Valor 2. Menciona el ahorro energético de forma significativa, mencionando o subrayando de alguna forma la relevancia.

6. Argumenta motivos morales y/o solidarios (Se mide en las preguntas 4 y 5.2).

Valor 1. No argumenta motivos morales o solidarios o argumenta motivos morales y/o solidarios pero de forma no significativa, sin énfasis o apoyo explícito.

Valor 2. Argumenta motivos morales y/o solidarios de forma significativa, mencionando o subrayando de alguna forma la relevancia.

7. Se implica personalmente (Se mide en las preguntas 5.1 y 5.2).

Valor 1. No menciona argumentos que indiquen una implicación personal o menciona argumentos que indican una implicación personal pero de forma no significativa, sin énfasis o apoyo explícito.

Valor 2. Menciona argumentos que indican una implicación personal de forma significativa, mencionando o subrayando de alguna forma la relevancia.

Tras realizar el análisis de los chi cuadrado en los datos obtenidos,en los cuales se consideró un nivel de confianza del 0,05 (95\%) y dos grados de libertad, observamos los siguientes resultados, donde observamos, para cada parámetro medido en las diferentes cuestiones, si es significativa la diferencia encontrada entre realizar el juego de rol o no realizarlo para las respuestas dadas:

\begin{tabular}{|c|c|c|}
\hline \multicolumn{2}{|r|}{ Parámetro medido: Argumenta motivos ambientales } & \\
\hline $\begin{array}{ll}\text { Pregunta en } \\
\text { la que } \\
\text { analiza }\end{array}$ & $\begin{array}{l}\text { ¿Es significativa la diferencia entre realizar el juego de rol o no } \\
\text { realizarlo? }\end{array}$ & $\begin{array}{l}\text { Valor } \chi^{2} \\
\text { obtenido }\end{array}$ \\
\hline 1 & No & 2,97 \\
\hline 2 & No & 1,62 \\
\hline
\end{tabular}

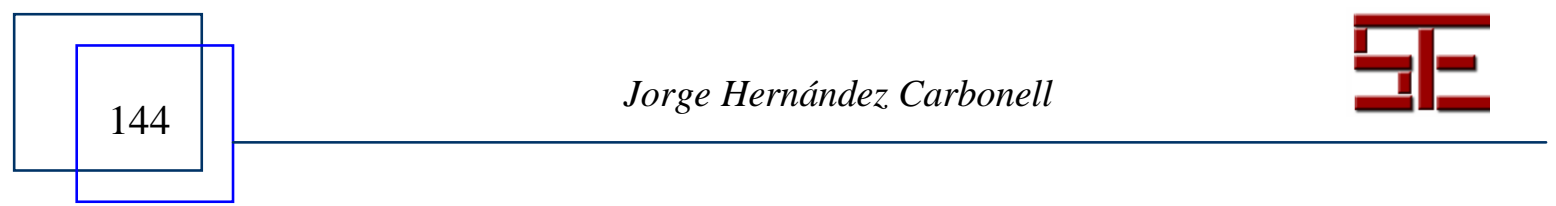




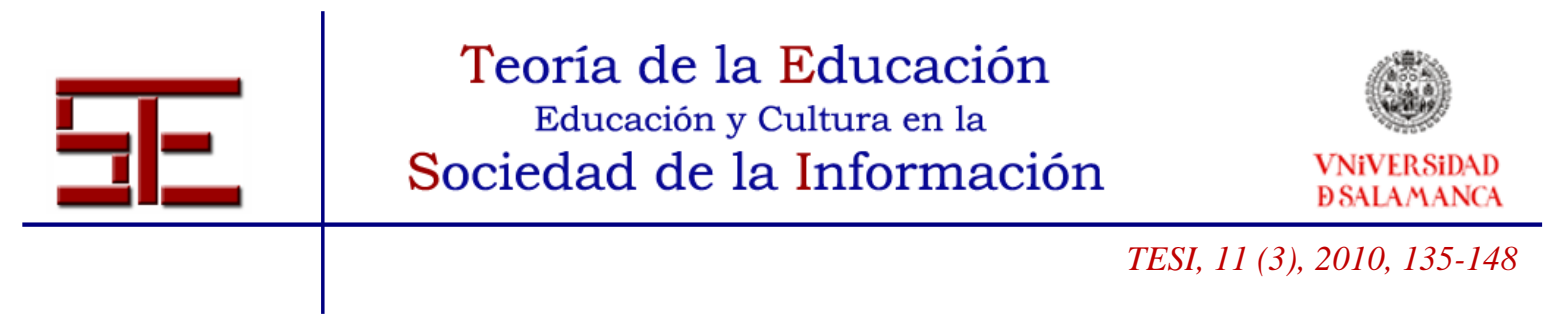

\begin{tabular}{|c|c|c|}
\hline 3 & No & 3,74 \\
\hline 4 & No & 3,92 \\
\hline
\end{tabular}

\begin{tabular}{|cr|c|c|}
\hline \multicolumn{3}{|c|}{ Parámetro medido: Actitud ante la limitación de recursos energéticos } & \\
\hline $\begin{array}{l}\text { Pregunta en } \\
\text { la que } \\
\text { analiza }\end{array}$ & se & $\begin{array}{l}\text { iEs significativa la diferencia entre realizar el juego de rol o no } \\
\text { realizarlo? }\end{array}$ & $\begin{array}{c}\text { Valor } \chi^{2} \\
\text { obtenido }\end{array}$ \\
\hline 1 & & $\mathrm{Si}$ & 14,68 \\
\hline 3 & $\mathrm{Si}$ & 11,59 \\
\hline 4 & $\mathrm{Si}$ & 27,66 \\
\hline
\end{tabular}

\begin{tabular}{|c|c|c|}
\hline \multicolumn{4}{|l|}{ Parámetro medido: Valora el medio ambiente como prioritario } \\
\hline $\begin{array}{l}\text { Pregunta en la } \\
\text { que se analiza }\end{array}$ & $\begin{array}{l}\text { ¿Es significativa la diferencia entre } \\
\text { realizar el juego de rol o no } \\
\text { realizarlo? }\end{array}$ & Valor $\chi^{2}$ obtenido \\
\hline 1 & No & 9,35 \\
\hline 2 & No & 9,35 \\
\hline 3 & No & 9,31 \\
\hline 4 & Si & 11,67 \\
\hline
\end{tabular}

\begin{tabular}{|c|c|c|}
\hline \multicolumn{3}{|c|}{ Parámetro medido: Actitud positiva ante las energías alternativas } \\
\hline $\begin{array}{c}\text { Pregunta en } \\
\text { la que se } \\
\text { analiza }\end{array}$ & $\begin{array}{c}\text { iEs significativa la diferencia } \\
\text { entre realizar el juego de rol o } \\
\text { no realizarlo? }\end{array}$ & Valor $\chi^{2}$ obtenido \\
\hline 1 & $\mathrm{Si}$ & 12,17 \\
\hline 2 & $\mathrm{Si}$ & 19,52 \\
\hline 3 & No & 9,35 \\
\hline 5.2 & No & 6,14 \\
\hline
\end{tabular}

\begin{tabular}{|c|c|c|}
\hline \multicolumn{2}{|c|}{ Parámetro medido: Valora el ahorro energético } & \multirow[b]{2}{*}{$\begin{array}{l}\text { Valor } \chi^{2} \\
\text { obtenido }\end{array}$} \\
\hline $\begin{array}{l}\text { Pregunta en } \\
\text { la que se } \\
\text { analiza }\end{array}$ & $\begin{array}{l}\text { ¿Es significativa la diferencia entre realizar el juego } \\
\text { de rol o no realizarlo? }\end{array}$ & \\
\hline 4 & $\mathrm{Si}$ & 10,88 \\
\hline 5.2 & $\mathrm{Si}$ & 9,27 \\
\hline
\end{tabular}

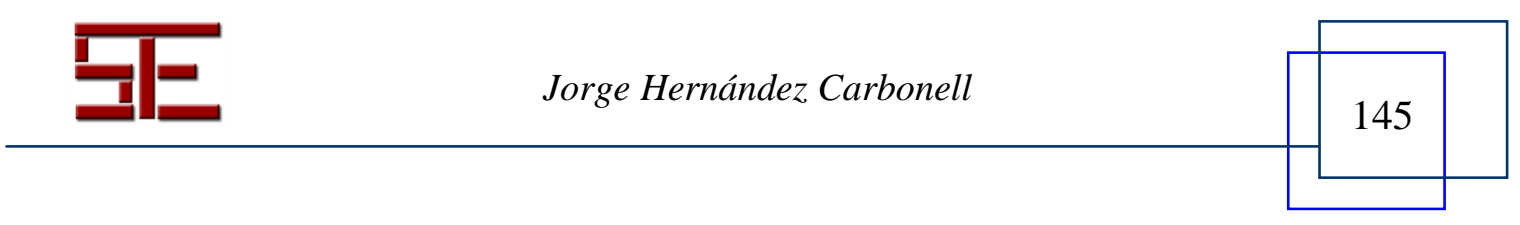




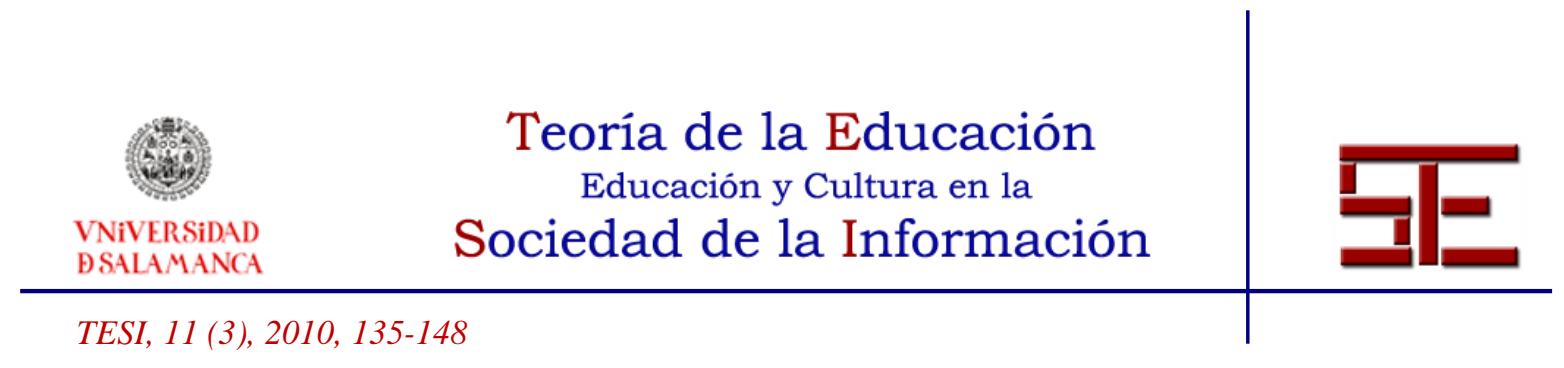

\begin{tabular}{|c|c|c|}
\hline \multicolumn{2}{|c|}{$\begin{array}{l}\text { Parámetro medido: } \\
\text { Argumenta motivos morales y/o solidarios }\end{array}$} & \multirow[b]{2}{*}{$\begin{array}{l}\text { Valor } \chi^{2} \\
\text { obtenido }\end{array}$} \\
\hline $\begin{array}{l}\text { Pregunta en la } \\
\text { que se analiza }\end{array}$ & $\begin{array}{l}\text { ¿Es significativa la diferencia entre realizar el juego de } \\
\text { rol o no realizarlo? }\end{array}$ & \\
\hline 4 & $\mathrm{Si}$ & 20,30 \\
\hline 5.2 & $\mathrm{Si}$ & 11,17 \\
\hline
\end{tabular}

\begin{tabular}{|c|l|l|c|}
\hline \multicolumn{2}{|l|}{ Parámetro medido: Se implica personalmente } & \\
\hline $\begin{array}{l}\text { Pregunta en } \\
\text { la que } \\
\text { analiza }\end{array}$ & $\begin{array}{l}\text { ¿Es significativa la diferencia entre realizar el juego de rol o } \\
\text { no realizarlo? }\end{array}$ & $\begin{array}{c}\text { Valor } \chi^{2} \\
\text { obtenido }\end{array}$ \\
\hline 5.1 & $\mathrm{Si}$ & 13,51 \\
\hline 5.2 & $\mathrm{Si}$ & 21,74 \\
\hline
\end{tabular}

\section{4.- CONCLUSIONES}

En los resultados anteriores, se observa que dependiendo del parámetro analizado la utilización del juego de rol será significativa o no en el tratamiento educativo analizado. Así, observamos que el cambio no es significativo en la argumentación de motivos ambientales. Ello significaría que utilizar el juego de rol o no utilizarlo produce efectos de cambio similares.

Observamos que el cambio es significativo en:

- Actitud ante la limitación de recursos energéticos

- Valora el medio ambiente como prioritario

- Actitud positiva ante las energías alternativas

- Valora el ahorro energético

- Argumenta motivos morales y/o solidarios

- Se implica personalmente

lo que implica, que la realización del juego de rol produce efectos de cambio superiores a los logrados si no se realiza el juego de rol.

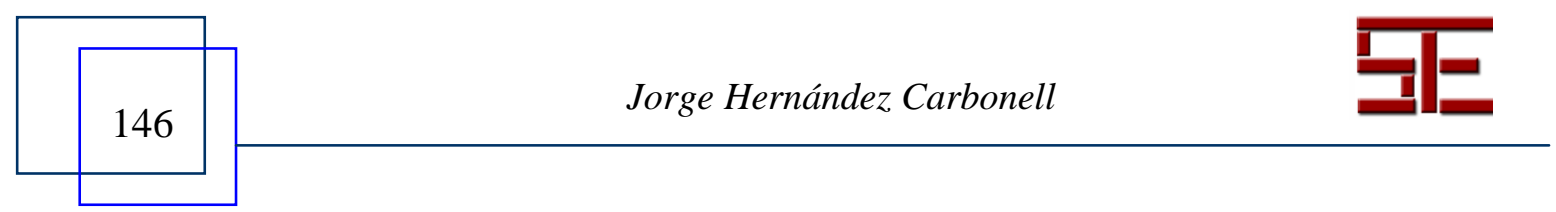




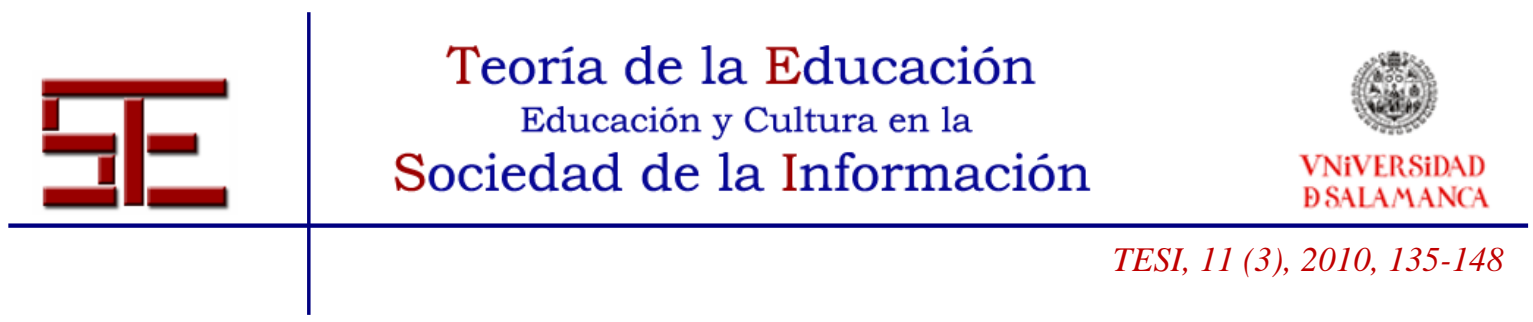

En lo que respecta a la valoración del medio ambiente como prioritario y actitud positiva ante las energías alternativas, se observa diferencia significativa en la mayoría de los casos, existiendo en algunos de ellos que no lo son.

Esto implicaría que el uso del juego de rol produce efectos de cambio superiores a los logrados sin el juego de rol, aunque en algunos casos es indiferente usar o no usar el juego de rol, ya que su utilización o no semana produce efectos de cambio similares en estos dos parámetros.

Se observa que en la actitud ante la limitación de recursos energéticos, la valoración del ahorro energético, la argumentación de motivos morales y/o solidarios y la implicación personal la diferencia es significativa en todos los casos, lo que implicaría que el uso del juego de rol produce efectos de cambio superiores a los logrados sin el juego de rol.

De los análisis anteriores podemos concluir que la utilización de los juegos de rol potencia los cambios en las actitudes y valores de los usuarios de estos juegos.

\section{5.- BIBLIOGRAFÍA.}

Acevedo, J. A. (2000). Algunas creencias sobre el conocimiento científico de los profesores de Educación Secundaria en formación inicial. Bordón, 52 (1), 5-16.

Aikenhead, G.S. (1988). An analysis of four ways of assessing student beliefs about STS topics. Journal of Research in Science Teaching, 25 (8), 607-629.

Caurin, C. (1999). Análisis, Evaluación y Modificación de Actitudes en Educación Ambiental. Tesis Doctoral. Universidad de Valencia. Publicaciones.

Caurín, C.; Gil, Mª \& Llopis, J. (2000). Analysis, Evaluation y Change of Attitudes in environmental Education: Is it posible? En Resumen de ponencias del Congreso de Didáctica de las Ciencias de Santiago de Compostela, 2000. Santiago de Compostela.

Escudero, T. (1985). Las actitudes en la enseñanza de las Ciencias: un panorama complejo. Revista de Educación, 278, 5-25.

Esteban, A. y Vecina, M. L. (2000). Síntesis del estudio sobre los efectos psicosociales de los juegos de rol en el desarrollo social y cognitivo de los menores. Boletín Oficial de la Asamblea de Madrid, $\mathrm{n}^{\circ}$ 46, 5024-5032.

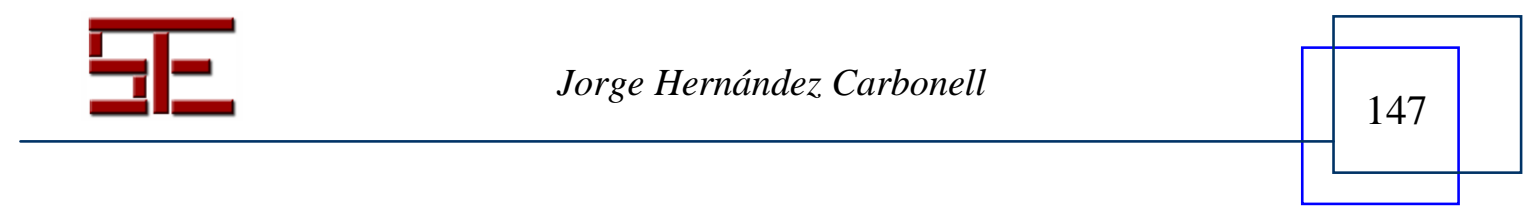




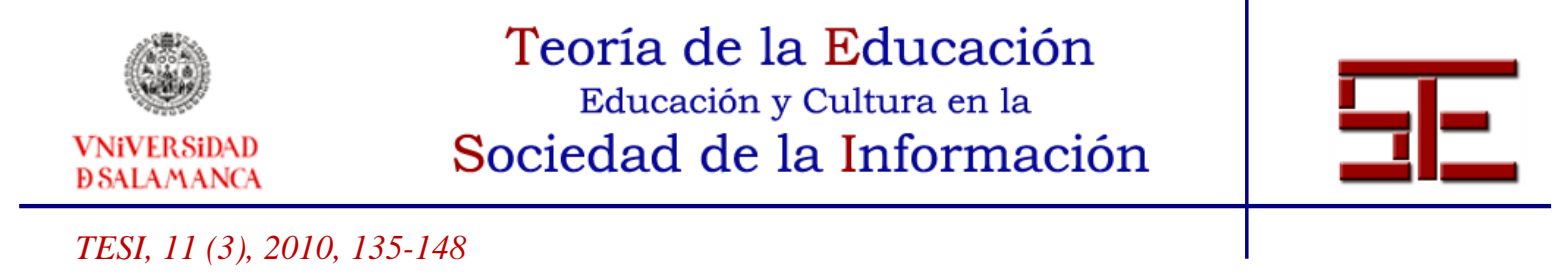

García, J y Nando, J. (2000). Estrategias didácticas en Educación ambiental. Málaga: Ediciones Aljibe.

Guilbert, L. \& Meloche, D. (1993). L’Idée de Science chez des enseignants en formation: Un lieu entre l'histoire des sciences et l'hétérogénéité des visions? Didas Kalia 2, 7-20.

Hernández, J. (2005). Educación de Actitudes y Valores en un contexto no Formal. La problemática de la energía. Tesis Doctoral. Universidad de Valencia. Publicaciones.

Sanmartí, N. (1989). Dificultats en la comprensió de la diferenciació entre els conceptes de mescla y compost. Tesis Doctoral. Universidad Autónoma de Barcelona.

Para citar el presente artículo puede utilizar la siguiente referencia:

Hernández Carbonell, J. (2010). Cambio de actitudes y valores ante la energía tras el uso de un juego de rol, en Orejudo González, J.P. (Coord.) Perspectiva educativa y cultural de "juego de rol". Revista Teoría de la Educación: Educación y Cultura en la Sociedad de la Información. Vol. 11, n 3. Universidad de Salamanca, pp. 135-148 [Fecha de consulta: $\mathrm{dd} / \mathrm{mm} / \mathrm{aaaa}$ ].

http://campus.usal.es/ revistas_trabajo/index.php/revistatesi/article/view/7452/7469 